This item was submitted to Loughborough's Research Repository by the author.

Items in Figshare are protected by copyright, with all rights reserved, unless otherwise indicated.

\title{
Applying coupon-collecting theory to computer-aided assessments
}

PLEASE CITE THE PUBLISHED VERSION

PUBLISHER

(c) Cambridge University Press

VERSION

AM (Accepted Manuscript)

LICENCE

CC BY-NC-ND 4.0

REPOSITORY RECORD

Goldie, Charles M., Rosie Cornish, and Carol L. Robinson. 2019. "Applying Coupon-collecting Theory to Computer-aided Assessments”. figshare. https://hdl.handle.net/2134/8781. 
This item was submitted to Loughborough's Institutional Repository (https://dspace.lboro.ac.uk/) by the author and is made available under the following Creative Commons Licence conditions.

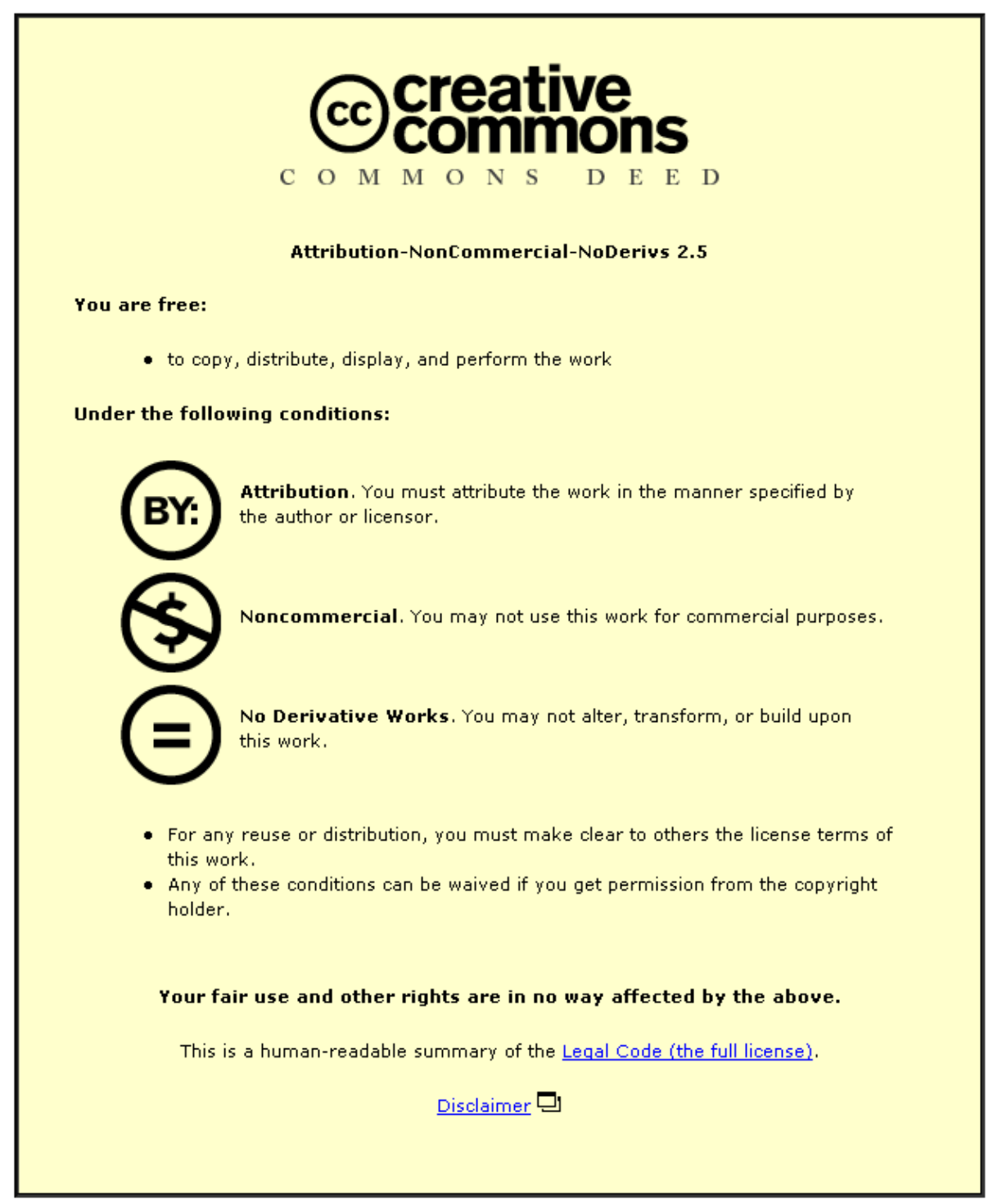

For the full text of this licence, please go to: http://creativecommons.org/licenses/by-nc-nd/2.5/ 


\title{
APPLYING COUPON-COLLECTING THEORY TO COMPUTER-AIDED ASSESSMENTS
}

\author{
C. M. GOLDIE, R. CORNISH, AND C. L. ROBINSON
}

\begin{abstract}
Computer-based tests with randomly generated questions allow a large number of different tests to be generated. Given a fixed number of alternatives for each question, the number of tests that need to be generated before all possible questions have appeared is surprisingly low.
\end{abstract}

\section{INTRODUCTION}

The use of computer-based tests in which questions are randomly generated in some way provides a means whereby a large number of different tests can be generated; many universities currently use such tests as part of the student assessment process. In this paper we present findings that illustrate that, although the number of different possible tests is high and grows very rapidly as the number of alternatives for each question increases, the average number of tests that need to be generated before all possible questions have appeared at least once is surprisingly low. We presented preliminary findings along these lines in $[5]$.

The problem is set out as follows. A computer-based test consists of $q$ questions, each (independently) selected at random from a separate bank of $a$ alternatives. Let $N_{q}$ be the number of tests one needs to generate in order to see all the $a q$ questions in the $q$ question banks at least once. We are interested in how, for fixed $a$, the random variable $N_{q}$ grows with the number of questions $q$ in the test. Typically, $a$ might be 10 - i.e. each question might have a bank of 10 alternatives - but we shall allow any value of $a$, and give numerical results for $a=20$ and $a=5$ as well as for $a=10$.

\section{Coupon collecting}

In the case $q=1$, i.e. a one-question test, we re-notate $N_{q}$ as $Y$, and observe that we have an equivalent to the classic coupon-collector problem: your favourite cereal has a coupon in each packet, and there are $a$ alternative types of coupon. $Y$ is the number of packets you have to buy in order to get at least one coupon of each of the $a$ types. The coupon-collector problem has been much studied; see e.g. Grimmett \& Stirzaker [7, p. 55]. 
We can write $Y$ as

$$
Y=Y_{1}+Y_{2}+\cdots+Y_{a}
$$

where each $Y_{i}$ is the number of cereal packets you must buy in order to acquire a new type of coupon, when you already have $i-1$ types in your collection. Thus $Y_{1}=1, Y_{2}$ is the number of further packets you find you need to gain a second type, and so on. The random variables $Y_{1}, \ldots, Y_{a}$ are mutually independent. For the distribution of $Y_{k}$, clearly

$$
P\left(Y_{k}=y\right)=\frac{a-k+1}{a}\left(\frac{k-1}{a}\right)^{y-1} \quad(y=1,2, \ldots) .
$$

We shall say that $X \sim \operatorname{Geom}(p)$, or $X$ has a geometric distribution with parameter $p$, if $P(X=x)=p(1-p)^{x-1}$ for $x=1,2, \ldots$ Thus $Y_{k} \sim \operatorname{Geom}((a-k+1) / a)$. As the $\operatorname{Geom}(p)$ distribution has expectation $1 / p$ it follows that

$$
E Y=\sum_{k=1}^{a} E Y_{k}=\sum_{k=1}^{a} \frac{a}{a-k+1}=a \sum_{k=1}^{a} \frac{1}{k} .
$$

For different values of $a$ we therefore have the following.

$$
\begin{array}{c|cccc}
a & 5 & 10 & 15 & 20 \\
E Y & 11 \cdot 42 & 29 \cdot 29 & 49 \cdot 77 & 71 \cdot 96
\end{array}
$$

In other words, if there are 10 coupons to collect then an average of 29 packets of cereal would have to be bought in order to obtain all 10 of these coupons. In the context of computer-based tests, if a test had one question selected at random from a bank of 10 alternatives, an average of 29 tests would need to be generated in order to see all the questions at least once.

To apply the theory to tests with more than one question we will also need an explicit expression for $P(Y>y)$. To revert to the language of coupons in cereal packets, let us number the coupon types $1,2, \ldots, a$, and let $A_{i}$ be the event that type $i$ does not occur in the first $y$ cereal packets bought. The event that $Y>y$ is then the union of the events $A_{1}, A_{2}, \ldots, A_{a}$. So by the inclusion-exclusion formula,

$$
\begin{aligned}
P(Y>y)= & P\left(\bigcup_{i=1}^{a} A_{i}\right) \\
= & \sum_{i=1}^{a} P\left(A_{i}\right)-\sum_{i<j} P\left(A_{i} \cap A_{j}\right)+\sum_{i<j<k} P\left(A_{i} \cap A_{j} \cap A_{k}\right)-\cdots \\
& \quad+(-1)^{a+1} P\left(A_{1} \cap \cdots \cap A_{a}\right) .
\end{aligned}
$$

Obviously $P\left(A_{i}\right)=(1-1 / a)^{y}$ for each $i$. For distinct $i$ and $j, A_{i} \cap A_{j}$ is the event that a particular two of the $a$ coupon types do not occur in the first $y$ purchases, so has probability $(1-2 / a)^{y}$. Similarly $A_{i} \cap A_{j} \cap A_{k}$, 
for distinct $i, j$ and $k$, has probability $(1-3 / a)^{y}$, and so on. We conclude that

$$
P(Y>y)=\sum_{k=1}^{a}(-1)^{k+1}\left(\begin{array}{l}
a \\
k
\end{array}\right)\left(1-\frac{k}{a}\right)^{y}
$$

(when $y>0$ the final term of the sum is zero). Let $F$ be the distribution function for $Y$; thus the above is equivalent to

$$
F(y):=P(Y \leq y)=\sum_{k=0}^{a}(-1)^{k}\left(\begin{array}{l}
a \\
k
\end{array}\right)\left(1-\frac{k}{a}\right)^{y} \quad(y=a, a+1, a+2, \ldots) .
$$

This is a classical formula for the probability that all cells are occupied when $y$ balls are distributed at random among a cells; cf. Feller [6, (11.11)]. The right-hand side of $(2.2)$ has value 0 when $y=0,1, \ldots$, $a-1$.

\section{How MAny Tests?}

We return to the initial question. We have a test containing $q$ questions, each selected at random from a bank of $a$ alternatives. $N_{q}$ is defined to be the number of tests that need to be generated in order to see all possible $a q$ questions at least once.

For question $j$ of the test, let $Y_{j}$ be the number of tests needed to see all the $a$ alternatives in its question bank. The random variables $Y_{1}, Y_{2}, \ldots, Y_{q}$ are mutually independent, each distributed as the $Y$ of the previous section, and $N_{q}$ is their maximum:

$$
N_{q}=\max \left\{Y_{1}, Y_{2}, \ldots, Y_{q}\right\} .
$$

We thus have

$$
\begin{aligned}
E N_{q} & =\sum_{n=0}^{\infty} P\left(N_{q}>n\right) \\
& =\sum_{n=0}^{\infty}\left(1-P\left(N_{q} \leq n\right)\right) \\
& =\sum_{n=0}^{\infty}\left(1-\prod_{j=1}^{q} P\left(Y_{j} \leq n\right)\right) \\
& =\sum_{n=0}^{\infty}\left(1-\prod_{j=1}^{q}\left(1-P\left(Y_{j}>n\right)\right)\right) \\
& =\sum_{n=0}^{\infty}\left(1-(1-P(Y>n))^{q}\right) .
\end{aligned}
$$


This can be reduced to a finite sum as follows.

$$
\begin{aligned}
E N_{q}= & \sum_{n=0}^{\infty} \sum_{m=1}^{q}(-1)^{m+1}\left(\begin{array}{c}
q \\
m
\end{array}\right)(P(Y>n))^{m} \\
= & \sum_{n=0}^{\infty} \sum_{m=1}^{q}(-1)^{m+1}\left(\begin{array}{c}
q \\
m
\end{array}\right) \sum_{j_{1}=1}^{a}(-1)^{j_{1}+1}\left(\begin{array}{c}
a \\
j_{1}
\end{array}\right)\left(1-\frac{j_{1}}{a}\right)^{n} \cdots \\
& \cdots \sum_{j_{m}=1}^{a}(-1)^{j_{m}+1}\left(\begin{array}{c}
a \\
j_{m}
\end{array}\right)\left(1-\frac{j_{m}}{a}\right)^{n} \\
=- & \sum_{m=1}^{q}\left(\begin{array}{c}
q \\
m
\end{array}\right) \sum_{j_{1}=1}^{a} \cdots \sum_{j_{m}=1}^{a}(-1)^{j_{1}+\cdots+j_{m}} \\
=- & \sum_{m=1}^{q}\left(\begin{array}{c}
a \\
j_{1}
\end{array}\right) \cdots\left(\begin{array}{c}
a \\
j_{m}
\end{array}\right) \sum_{n=0}^{\infty}\left(\prod_{i=1}^{m}\left(1-\frac{j_{i}}{a}\right)\right)^{n} \cdots \sum_{j_{1}=1}^{a} \frac{(-1)^{j_{1}+\cdots+j_{m}}\left(\begin{array}{c}
a \\
j_{1}
\end{array}\right) \cdots\left(\begin{array}{c}
a \\
j_{m}
\end{array}\right)}{1-\prod_{i=1}^{m}\left(1-j_{i} / a\right)} .
\end{aligned}
$$

This, though, is not well suited to computation, and we have used (3.1) for the numerical results below.

Note. The way in which CMG got involved in writing this paper was through chancing on a query posted by RC on Allstat, a UK-based electronic mailing list, asking how to calculate the expected number of tests a student would need to access in order to see the complete bank of questions. CMG immediately recognised the query as a form of coupon-collecting problem, but not quite in standard form. What he should have done then was to think and calculate, following Littlewood's famous advice [8, p. 93]

"It is of course good policy, and I have often practised it, to

begin without going too much into the existing literature".

What he actually did was to seek previous work using Google. With customary speed and accuracy, Google produced a list with paper [2] in position 6. Knowing that Sheldon Ross is unbeatable at combinatorial probability problems, CMG looked up this paper - and was thoroughly led astray. The paper does indeed treat our problem and is an excellent paper, but it is much more general than we needed and sets up a structure that obscures the relatively simple nature of what we needed for this problem. It was better to work the above out from first principles.

\section{Asymptotics}

We employ Extreme-Value Theory (EVT) to investigate the random variable $N_{q}$ as the number of questions $q$ becomes large, the number $a$ of alternatives per question staying fixed. It turns out we are in a case identified by C. W. Anderson in 1970, where a limit fails to exist but 
there are close bounds above and below. Thus despite the absence of a limit we gain asymptotic results of some precision.

The relevant extreme-value distribution will be the Gumbel distribution, with (cumulative) distribution function $\Lambda(x)=\exp \left(-e^{-x}\right)$ for all $x \in \mathbb{R}$; write $Z$ for a random variable with the Gumbel distribution.

Throughout this section $a \geq 2$ is an integer, and we set $\alpha:=$ $\log (a /(a-1))>0$. Proofs of the results in this section are in $\S 5$.

A first goal of EVT for the random variables $N_{q}$ would be to find a norming sequence $a_{q}>0$ and a centring sequence $b_{q}$ such that $\left(N_{q}-\right.$ $\left.b_{q}\right) / a_{q}$ has a limit distribution as $q \rightarrow \infty$.

Theorem 4.1. There do not exist sequences $a_{q}>0$ and $b_{q}$ such that $\left(N_{q}-b_{q}\right) / a_{q}$ has a non-degenerate limit distribution as $q \rightarrow \infty$. However, with $b_{q}:=\alpha^{-1} \log (a q)$ we have for all $x \in \mathbb{R}$ that

$$
\begin{aligned}
\Lambda(\alpha(x-1))=\liminf _{q \rightarrow \infty} P( & \left.N_{q}-b_{q} \leq x\right) \\
& \leq \limsup _{q \rightarrow \infty} P\left(N_{q}-b_{q} \leq x\right)=\Lambda(\alpha x) .
\end{aligned}
$$

Thus $N_{q}-b_{q}$, in distribution, is asymptotically between $\alpha^{-1} Z$ and $1+$ $\alpha^{-1} Z$, with $Z$ Gumbel, and these distributional bounds are sharp.

To describe the local behaviour, let $\lfloor x\rfloor$ denote the integer part of $x$, $\{x\}:=x-\lfloor x\rfloor$ the fractional part, and let $\lceil x\rceil:=\lfloor x\rfloor+1$. Then for each integer $n$,

$$
\begin{array}{r}
P\left(N_{q}-\left\lceil b_{q}\right\rceil=n\right)-\Lambda\left(\alpha\left(n+1-\left\{b_{q}\right\}\right)\right)+\Lambda\left(\alpha\left(n-\left\{b_{q}\right\}\right)\right) \rightarrow 0 \\
\text { as } q \rightarrow \infty .
\end{array}
$$

We remark that the Gumbel distribution has mean $\gamma \bumpeq 0.5771$, the Euler-Mascheroni constant, and variance $\pi^{2} / 6$. Its distribution tails decay exponentially or better: $\lim _{x \rightarrow \infty} e^{x}(1-\Lambda(x))=1$ and $\lim _{x \rightarrow-\infty} e^{-x} \Lambda(x)=0$. We use these facts below. We first extend the above stochastic boundedness of the sequence $\left(N_{q}-b_{q}\right)$ to $L_{p}$ boundedness for all $p$. For the rest of the paper we set $b_{q}:=\alpha^{-1} \log (a q)$ and $R_{q}:=N_{q}-b_{q}$.

Theorem 4.2. For each $p \geq 1, \sup _{q \in \mathbb{N}} E\left(\left|R_{q}\right|^{p}\right)<\infty$.

Theorem 4.2 implies that the distributional asymptotics of Theorem 4.1 will extend to give asymptotic bounds on moments. Moment convergence in EVT is treated in Resnick $[9, \S 2.1]$, and we use some of the ideas from the proofs there in proving the results below.

\section{Theorem 4.3.}

$$
\begin{aligned}
\frac{\gamma+\log a}{\alpha} \leq \liminf _{q \rightarrow \infty}\left(E N_{q}\right. & \left.-\frac{\log q}{\alpha}\right) \\
& \leq \limsup _{q \rightarrow \infty}\left(E N_{q}-\frac{\log q}{\alpha}\right) \leq \frac{\gamma+\log a}{\alpha}+1 .
\end{aligned}
$$


By similar methods one may obtain bounds on higher moments. We content ourselves with those on the second moment, leading to good bounds on var $N_{q}$, the variance of $N_{q}$.

Lemma 4.4.

$$
\begin{aligned}
& E\left(\left(1+\alpha^{-1} Z\right)^{2} \mathbf{1}_{1+\alpha^{-1} Z \leq 0}+\left(\alpha^{-1} Z\right)^{2} \mathbf{1}_{Z>0}\right) \leq \liminf _{q \rightarrow \infty} E\left(R_{q}^{2}\right) \\
& \leq \limsup _{q \rightarrow \infty} E\left(R_{q}^{2}\right) \leq E\left(\left(\alpha^{-1} Z\right)^{2} \mathbf{1}_{Z \leq 0}+\left(1+\alpha^{-1} Z\right)^{2} \mathbf{1}_{1+\alpha^{-1} Z>0}\right) .
\end{aligned}
$$

\section{Theorem 4.5.}

$$
\limsup _{q \rightarrow \infty}\left|\operatorname{var} N_{q}-\frac{\pi^{2}}{6 \alpha^{2}}\right| \leq \theta(\alpha)+1-e^{-1}+\frac{2\left(\gamma+E_{1}(1)\right)}{\alpha},
$$

where $\theta(\alpha)=E\left(\left(1+\alpha^{-1} Z\right)^{2} \mathbf{1}_{0<1+\alpha^{-1} Z \leq 1}\right)$ satisfies $0<\theta(\alpha)<1$, and $E_{1}(1)=\int_{1}^{\infty} t^{-1} e^{-t} d t \bumpeq 0 \cdot 2194$.

Here, $E_{1}(1)$ is a value of the exponential integral (cf. Abramowitz \& Stegun $[1, \S 5.1]) E_{n}(x)=\int_{1}^{\infty} t^{-n} e^{-x t} d t$.

\section{PROOFS FOR $\S 4$}

Proof of Theorem 4.1. In (2.1) the $k=1$ term dominates for large $y$, So

$$
P(Y>y)=a(1-1 / a)^{y}(1+\mathrm{o}(1))
$$

as $y \rightarrow \infty$ through integer values. As noted in Anderson [3, §1], the fact that the integer-valued random variable $Y$ has

$$
\frac{P(Y>y)}{P(Y>y+1)} \rightarrow \frac{a}{a-1}>1 \quad \text { as } y \rightarrow \infty
$$

prevents it from belonging to the 'domain of attraction' for maxima of any extreme-value distribution, and so no non-trivial limit distribution for $\left(N_{q}-b_{q}\right) / a_{q}$, for any choices of $a_{q}$ and $b_{q}$, can exist.

For the rest of the proof, $b_{q}:=\alpha^{-1} \log (a q)$. Via the definition of $\alpha$, (5.1) gives that $F(y)=1-a e^{-\alpha y}(1+\mathrm{o}(1))$ as $y \rightarrow \infty$ through integer values. So for each fixed $x \in \mathbb{R}$,

$$
P\left(N_{q}-b_{q} \leq x\right)=F^{q}\left(\left\lfloor x+b_{q}\right\rfloor\right)=\left(1-a e^{-\alpha\left\lfloor x+b_{q}\right\rfloor}(1+\mathrm{o}(1))\right)^{q}
$$

as $q \rightarrow \infty$. Then

$$
\begin{aligned}
P\left(N_{q}-b_{q} \leq x\right) & \leq\left(1-a e^{-\alpha\left(x+b_{q}\right)}(1+\mathrm{o}(1))\right)^{q} \\
& =\left(1-\frac{e^{-\alpha x}(1+\mathrm{o}(1))}{q}\right)^{q} \rightarrow \Lambda(\alpha x) \quad \text { as } q \rightarrow \infty .
\end{aligned}
$$

With $x \in \mathbb{R}$ still fixed we define the sequence $(q(k))_{k=1}^{\infty}$ to be those $q$ for which the interval $\left(x+b_{q-1}, x+b_{q}\right]$ contains one or more integers, i.e. for which $x+b_{q-1}<\left\lfloor x+b_{q}\right\rfloor$. Since $b_{q} \rightarrow \infty$ this is an infinite sequence, and since $b_{q+1}-b_{q} \rightarrow 0$ we have $x+b_{q(k)}-\left\lfloor x+b_{q(k)}\right\rfloor \rightarrow 0$ as $k \rightarrow \infty$, 
whence with (5.2) we conclude that $P\left(N_{q(k)}-b_{q(k)} \leq x\right) \rightarrow \Lambda(\alpha x)$ as $k \rightarrow \infty$. Thus $\lim \sup _{q \rightarrow \infty} P\left(N_{q}-b_{q} \leq x\right)=\Lambda(\alpha x)$.

For the limit inferior,

$$
\begin{aligned}
P\left(N_{q}-b_{q} \leq x\right) & \geq\left(1-a e^{-\alpha\left(x-1+b_{q}\right)}(1+\mathrm{o}(1))\right)^{q} \\
& =\left(1-\frac{e^{-\alpha(x-1)}(1+\mathrm{o}(1))}{q}\right)^{q} \\
& \rightarrow \Lambda(\alpha(x-1)) \quad \text { as } q \rightarrow \infty
\end{aligned}
$$

With the same sequence $(q(k))$ as above, note that $x+b_{q(k)-1}-\lfloor x+$ $\left.b_{q(k)-1}\right\rfloor \rightarrow 1$ as $k \rightarrow \infty$, so

$$
\begin{aligned}
P\left(N_{q(k)-1}-b_{q(k)-1} \leq x\right) & =\left(1-a e^{-\alpha\left\lfloor x+b_{q(k)-1}\right\rfloor}(1+\mathrm{o}(1))\right)^{q(k)-1} \\
& =\left(1-a e^{-\alpha\left(x+b_{q(k)-1}-1\right)}(1+\mathrm{o}(1))\right)^{q(k)-1}
\end{aligned}
$$

by (5.2). The right-hand side converges to $\Lambda(\alpha(x-1))$. Thus $\lim \inf _{q \rightarrow \infty}$ $P\left(N_{q}-b_{q} \leq x\right)=\Lambda(\alpha(x-1))$. This establishes (4.1).

The extension to local behaviour is due to Anderson [4]. To gain the conclusion as we formulate it, (4.2), we may argue directly: fix an integer $n$ and start from

$$
P\left(N_{q}-\left\lceil b_{q}\right\rceil \leq n\right)=F^{q}\left(n+\left\lceil b_{q}\right\rceil\right)=\left(1-a e^{-\alpha\left(n+\left\lceil b_{q}\right\rceil\right)}(1+\mathrm{o}(1))\right)^{q}
$$

as $q \rightarrow \infty$. Now

$$
a e^{-\alpha\left(n+\left\lceil b_{q}\right\rceil\right)}=\frac{e^{-\alpha\left(n+\left\lceil b_{q}\right\rceil-b_{q}\right)}}{q}=\frac{e^{-\alpha\left(n+1-\left\{b_{q}\right\}\right)}}{q},
$$

and as the convergence in $(1-c / q)^{q} \rightarrow e^{-c}$ is locally uniform in $c$ we deduce that

$$
P\left(N_{q}-\left\lceil b_{q}\right\rceil \leq n\right)-\Lambda\left(\alpha\left(n+1-\left\{b_{q}\right\}\right)\right) \rightarrow 0 \quad \text { as } q \rightarrow \infty .
$$

Subtract from this the corresponding formula with $n$ replaced by $n-1$, and (4.2) follows.

For the next result we need a uniform bound on expressions of the form $1-(1-u / n)^{n}$ :

Lemma 5.1. For any $u_{0}>0$ there exists a positive integer $n_{1}=n_{1}\left(u_{0}\right)$ such that for $n \geq n_{1}$ and $0 \leq u \leq u_{0}$,

$$
1-\left(1-\frac{u}{n}\right)^{n} \leq 2 u \text {. }
$$

Proof. There exists $t_{0}>0$ (its value is about $\left.0 \cdot 7968\right)$ such that $\log (1-$ $\left.t_{0}\right)=-2 t_{0}$, so $\log (1-t) \geq-2 t$ for $0 \leq t \leq t_{0}$. Take $n_{1} \geq u_{0} / t_{0}$, then $1-(1-u / n)^{n} \leq 1-e^{-2 u}$ for $n \geq n_{1}$ and $0 \leq u \leq u_{0}$, and as $1-e^{-2 u} \leq 2 u$ the result follows. 
Proof of Theorem 4.2. We write $\mathbf{1}_{T}:=1$ if statement $T$ is true, $\mathbf{1}_{T}:=0$ if $T$ is false. Fix $n \in \mathbb{N}$. The distribution of $N_{q}$ is such that $E\left(R_{q}^{2 n}\right)<\infty$ for all $q$. We prove that $\sup _{q \in \mathbb{N}} E\left(R_{q}^{2 n}\right)<\infty$. Now

$$
E\left(R_{q}^{2 n}\right)=\int_{(-\infty, 0]} x^{2 n} d P\left(R_{q} \leq x\right)-\int_{(0, \infty)} x^{2 n} d P\left(R_{q}>x\right),
$$

and so, on integrating by parts,

$$
\begin{aligned}
E\left(R_{q}^{2 n}\right) & =-2 n \int_{-\infty}^{0} x^{2 n-1} P\left(R_{q} \leq x\right) d x+2 n \int_{0}^{\infty} x^{2 n-1} P\left(R_{q}>x\right) d x \\
& =: A+B
\end{aligned}
$$

say.

In (2.1) the right-hand side is asymptotic to its first term, $a e^{-\alpha y}$. There exists $y_{0}$ such that for real $y \geq y_{0}$ (not just integer $\left.y\right), P(Y>$ $y) \leq 2 a e^{-\alpha(y-1)}$. So for $x \geq 0$ and $q \geq a^{-1} e^{\alpha y_{0}}$,

$$
P\left(Y>x+b_{q}\right) \leq 2 a e^{-\alpha\left(x+b_{q}-1\right)}=\frac{2}{q} e^{\alpha-\alpha x},
$$

and hence

$$
P\left(R_{q}>x\right)=1-\left(1-P\left(Y>x+b_{q}\right)\right)^{q} \leq 1-\left(1-\frac{2}{q} e^{\alpha-\alpha x}\right)^{q} .
$$

Now apply Lemma 5.1. It follows that there exists $q_{1}$ such that for $q \geq q_{1}$ and $x \geq 0$

$$
P\left(R_{q}>x\right) \leq 4 e^{\alpha-\alpha x}
$$

Therefore, for $q \geq q_{1}$,

$$
B=2 n \int_{0}^{\infty} x^{2 n-1} P\left(R_{q}>x\right) d x \leq 8 n \int_{0}^{\infty} x^{2 n-1} e^{\alpha-\alpha x} d x<\infty .
$$

It remains to bound $A$. Returning again to (2.1), observe that we may find $y_{1}$ so that $P(Y>y) \geq \frac{1}{2} a e^{-\alpha y}$ for all real $y \geq y_{1}$. Therefore for $x \geq y_{1}-b_{q}$ we have

$$
P\left(Y>x+b_{q}\right) \geq \frac{1}{2} a e^{-\alpha\left(x+b_{q}\right)}=\frac{1}{2 q} e^{-\alpha x},
$$

and so

$$
\begin{aligned}
P\left(R_{q} \leq x\right) & =\left(1-P\left(Y>x+b_{q}\right)\right)^{q} \\
& \leq \exp \left(-q P\left(Y>x+b_{q}\right)\right) \\
& \leq \exp \left(-\frac{1}{2} e^{-\alpha x}\right) \quad \text { for } x \geq y_{1}-b_{q} .
\end{aligned}
$$

In $A=-2 n \int_{-\infty}^{0} x^{2 n-1} P\left(R_{q} \leq x\right) d x$, the lower endpoint of the interval of integration may be taken to be $-b_{q}$, as the integrand vanishes 
below this point, and we then choose further to split the integral to obtain

$$
\begin{aligned}
A & =-2 n \int_{y_{1}-b_{q}}^{0} x^{2 n-1} P\left(R_{q} \leq x\right) d x-2 n \int_{-b_{q}}^{y_{1}-b_{q}} x^{2 n-1} P\left(R_{q} \leq x\right) d x \\
& =: A_{1}+A_{2},
\end{aligned}
$$

say. If we take $q$ so large that $b_{q}>y_{1},(5.3)$ gives

$$
\begin{aligned}
A_{1} & \leq-\int_{y_{1}-b_{q}}^{0} x^{2 n-1} \exp \left(-\frac{1}{2} e^{-\alpha x}\right) d x \\
& <-2 n \int_{-\infty}^{0} x^{2 n-1} \exp \left(-\frac{1}{2} e^{-\alpha x}\right) d x<\infty
\end{aligned}
$$

Finally,

$$
\begin{aligned}
A_{2} & =-2 n \int_{b_{q}}^{y_{1}-b_{q}} x^{2 n-1} F^{q}\left(x+b_{q}\right) d x \\
& =-2 n \int_{0}^{y_{1}}\left(u-b_{q}\right)^{2 n-1} F^{q}(u) d u \\
& \leq 2 n y_{1} b_{q}^{2 n-1} F^{q}\left(y_{1}\right) \\
& =2 n y_{1}\left(\frac{\log (a q)}{\alpha}\right)^{2 n-1} F^{q}\left(y_{1}\right) .
\end{aligned}
$$

This tends to 0 as $q \rightarrow \infty$, because $0<F\left(y_{1}\right)<1$.

We have shown that $\lim \sup _{q \rightarrow \infty} E\left(R_{q}^{2 n}\right)<\infty$, so $\sup _{q \in \mathbb{N}} E\left(R_{q}^{2 n}\right)<$ $\infty$ as claimed, and the result follows.

Before proving Theorem 4.3 we note that (4.1) says that for each $x \in \mathbb{R}$,

$$
\Lambda(\alpha(x-1))=\liminf _{q \rightarrow \infty} P\left(R_{q} \leq x\right) \leq \limsup _{q \rightarrow \infty} P\left(R_{q} \leq x\right)=\Lambda(\alpha x),
$$

and that what we have to prove is

$$
E\left(\alpha^{-1} Z\right) \leq \liminf _{q \rightarrow \infty} E R_{q} \leq \limsup _{q \rightarrow \infty} E R_{q} \leq E\left(1+\alpha^{-1} Z\right) .
$$

We use (5.4) mostly in the form

$$
\begin{aligned}
P\left(\alpha^{-1} Z>x\right)=\liminf _{q \rightarrow \infty} & P\left(R_{q}>x\right) \\
& \leq \limsup _{q \rightarrow \infty} P\left(R_{q}>x\right)=P\left(1+\alpha^{-1} Z>x\right)
\end{aligned}
$$

obtained by subtracting each component from 1 . We make much use of Fatou's Lemma, that for non-negative $f_{n}$,

$$
\liminf _{n \rightarrow \infty} \int f_{n} \geq \int \liminf _{n \rightarrow \infty} f_{n}
$$


and also of its extended form: that if $f_{n} \leq f$ and $f$ is integrable then

$$
\limsup _{n \rightarrow \infty} \int f_{n} \leq \int \limsup _{n \rightarrow \infty} f_{n}
$$

The latter may be deduced from the former by considering $f-f_{n}$.

Proof of Theorem 4.3. We use the fact that for a random variable $X$ with finite mean, and any constant $c$,

$$
E\left(X \mathbf{1}_{X>-c}\right)=-c P(X>-c)+\int_{-c}^{\infty} P(X>x) d x
$$

as may be proved by integrating $\int_{(-c, \infty)} x d P(X \leq x)$ by parts. We thus have, for $c>0$,

$$
\begin{aligned}
E R_{q} & \leq E\left(R_{q} \mathbf{1}_{R_{q}>-c}\right) \\
& =-c P\left(R_{q}>-c\right)+\int_{-c}^{c} P\left(R_{q}>x\right) d x+\int_{c}^{\infty} P\left(R_{q}>x\right) d x \\
& =: A+B+C,
\end{aligned}
$$

say. First, by the left-hand equality in (5.6), $\limsup _{q \rightarrow \infty} A=$ $-c P\left(\alpha^{-1} Z>-c\right)$. Second, from the right-hand equality in (5.6), and the extended Fatou Lemma (take the dominating integrable function to be 1),

$$
\limsup _{q \rightarrow \infty} B \leq \int_{-c}^{c} P\left(1+\alpha^{-1} Z>x\right) d x \leq \int_{-c}^{\infty} P\left(1+\alpha^{-1} Z>x\right) d x .
$$

Combining the bounds on $A$ and $B$ yields

$$
\begin{aligned}
& \limsup _{q \rightarrow \infty}(A+B) \leq- c P\left(1+\alpha^{-1} Z>-c\right)+\int_{-c}^{\infty} P\left(1+\alpha^{-1} Z>x\right) d x \\
&+c\left(P\left(1+\alpha^{-1} Z>-c\right)-P\left(\alpha^{-1} Z>-c\right)\right) \\
&=E\left(\left(1+\alpha^{-1} Z\right) \mathbf{1}_{1+\alpha^{-1} Z>-c}\right) \\
& \quad+c P\left(-c-1<\alpha^{-1} Z \leq-c\right) \\
&<E\left(\left(1+\alpha^{-1} Z\right) \mathbf{1}_{1+\alpha^{-1} Z>-c}\right)+c P\left(\alpha^{-1} Z \leq-c\right) .
\end{aligned}
$$

For the third upper bound, on $C$, we note (with an eye to the next proof as well) that by Theorem $4.2, K:=\sup _{q \in \mathbb{N}} E\left(\left|R_{q}\right|^{3}\right)<\infty$. Then for $x>0, P\left(R_{q}>x\right) \leq K / x^{3}$, hence $C \leq K /\left(2 c^{2}\right)$. On combining this bound with that on $A+B$ we gain an upper bound on $\lim \sup _{q \rightarrow \infty} E R_{q}$ that converges to $E\left(1+\alpha^{-1} Z\right)$ as $c \rightarrow \infty$, concluding the proof of the upper bound in (5.5). 
For the lower bound we again use (5.7), this time to write

$$
\begin{aligned}
E R_{q} & =E\left(R_{q} \mathbf{1}_{R_{q} \leq-c}\right)+E\left(R_{q} \mathbf{1}_{R_{q}>-c}\right) \\
& =E\left(R_{q} \mathbf{1}_{R_{q} \leq-c}\right)-c P\left(R_{q}>-c\right)+\int_{-c}^{\infty} P\left(R_{q}>x\right) d x \\
& =: \tilde{A}+\tilde{B}+\tilde{C},
\end{aligned}
$$

say. First, Fatou's Lemma and then the left-hand equality in (5.6) give

$$
\liminf _{q \rightarrow \infty} \tilde{C} \geq \int_{-c}^{\infty} \liminf _{q \rightarrow \infty} P\left(R_{q}>x\right) d x=\int_{-c}^{\infty} P\left(\alpha^{-1} Z>x\right) d x .
$$

Second,

$$
\liminf _{q \rightarrow \infty} \tilde{B}=-c \limsup _{q \rightarrow \infty} P\left(R_{q}>-c\right)=-c P\left(1+\alpha^{-1} Z>-c\right),
$$

this time by the right-hand equality in (5.6). Combining, we find that

$$
\begin{aligned}
\liminf _{q \rightarrow \infty}(\tilde{B}+\tilde{C}) \geq & -c P\left(\alpha^{-1} Z>-c\right)+\int_{-c}^{\infty} P\left(\alpha^{-1} Z>x\right) d x \\
& -c\left(P\left(1+\alpha^{-1} Z>-c\right)-P\left(\alpha^{-1} Z>-c\right)\right) \\
= & E\left(\alpha^{-1} Z \mathbf{1}_{\alpha^{-1} Z>-c}\right)-c P\left(-c-1<\alpha^{-1} Z \leq-c\right) \\
\geq & E\left(\alpha^{-1} Z\right)-c P\left(\alpha^{-1} Z \leq-c\right) .
\end{aligned}
$$

Finally, to put a lower bound on $\tilde{A}$ we may again use the 'Markov inequality' method used above for $C$, obtaining $\tilde{A} \geq-K /\left(2 c^{2}\right)$. Combining this with the above, we gain a lower bound on $\lim _{i n f} E R_{q}$ that converges to $E\left(\alpha^{-1} Z\right)$ as $c \rightarrow \infty$. We thus obtain the lower bound in (5.5).

Proof of Lemma 4.4. We use variants of the decompositions in the previous proof. First, the upper bound. With $c>0$ fixed,

$$
\begin{aligned}
E\left(R_{q}^{2}\right)= & E\left(R_{q}^{2} \mathbf{1}_{R_{q}>-c}\right)+E\left(R_{q}^{2} \mathbf{1}_{R_{q} \leq-c}\right) \\
= & c^{2} P\left(R_{q}>-c\right)+2 \int_{-c}^{\infty} x P\left(R_{q}>x\right) d x+E\left(R_{q}^{2} \mathbf{1}_{R_{q} \leq-c}\right) \\
= & c^{2} P\left(R_{q}>-c\right)+2 \int_{-c}^{0} x P\left(R_{q}>x\right) d x+2 \int_{0}^{c} x P\left(R_{q}>x\right) d x \\
& \quad+2 \int_{c}^{\infty} x P\left(R_{q}>x\right) d x+E\left(R_{q}^{2} \mathbf{1}_{R_{q} \leq-c}\right) \\
= & : A+B_{1}+B_{2}+C+D,
\end{aligned}
$$

say. By the right-hand equality in (5.6), $\limsup _{q \rightarrow \infty} A=c^{2} P(1+$ $\left.\alpha^{-1} Z>-c\right)$. By the left-hand equality and Fatou's Lemma, followed 
by an integration by parts,

$$
\begin{aligned}
\limsup _{q \rightarrow \infty} B_{1} & \leq 2 \int_{-c}^{0} x P\left(\alpha^{-1} Z>x\right) d x \\
& =-c^{2} P\left(\alpha^{-1} Z>-c\right)+E\left(\left(\alpha^{-1} Z\right)^{2} \mathbf{1}_{-c<\alpha^{-1} Z \leq 0}\right) .
\end{aligned}
$$

Combining,

$$
\begin{aligned}
\limsup _{q \rightarrow \infty}\left(A+B_{1}\right) \leq & c^{2} P\left(-c-1<\alpha^{-1} Z \leq-c\right) \\
& \quad+E\left(\left(\alpha^{-1} Z\right)^{2} \mathbf{1}_{-c<\alpha^{-1}} Z \leq 0\right) \\
\leq & c^{2} P\left(\alpha^{-1} Z \leq-c\right)+E\left(\left(\alpha^{-1} Z\right)^{2} \mathbf{1}_{Z \leq 0}\right) .
\end{aligned}
$$

Next, by the right-hand equality in (5.6), and the extended Fatou Lemma,

$$
\begin{aligned}
\limsup _{q \rightarrow \infty} B_{2} & \leq 2 \int_{0}^{c} x P\left(1+\alpha^{-1} Z>x\right) d x \\
& \leq 2 \int_{0}^{\infty} x P\left(1+\alpha^{-1} Z>x\right) d x \\
& =E\left(\left(1+\alpha^{-1} Z\right)^{2} \mathbf{1}_{1+\alpha^{-1} Z>0}\right) .
\end{aligned}
$$

On combining this with (5.8) and letting $c \rightarrow \infty$ we conclude that

$$
\begin{aligned}
\lim _{c \rightarrow \infty} \limsup _{q \rightarrow \infty}\left(A+B_{1}\right. & \left.+B_{2}\right) \\
& \leq E\left(\left(\alpha^{-1} Z\right)^{2} \mathbf{1}_{Z \leq 0}+\left(1+\alpha^{-1} Z\right)^{2} \mathbf{1}_{1+\alpha^{-1} Z>0}\right) .
\end{aligned}
$$

The upper bound in (4.3) will follow if we can show that $\lim _{c \rightarrow \infty}$ $\limsup _{q \rightarrow \infty} C=0$, and likewise for $D$. For $C$ this follows by inserting into its defining formula the bound $P\left(R_{q}>x\right) \leq K / x^{3}$ developed in the proofs above, while for $D$ it follows from Theorem 4.2 via the uniform integrability of the family $\left(R_{q}^{2}\right)_{q \in \mathbb{N}}$. The upper bound in (4.3) is proved.

For the lower bound we fix $c>0$ and write

$$
\begin{aligned}
E\left(R_{q}^{2}\right) & \geq E\left(R_{q}^{2} \mathbf{1}_{R_{q}>-c}\right) \\
& =c^{2} P\left(R_{q}>-c\right)+2 \int_{-c}^{\infty} x P\left(R_{q}>x\right) d x \\
& \geq c^{2} P\left(R_{q}>-c\right)+2 \int_{-c}^{0} x P\left(R_{q}>x\right) d x+2 \int_{0}^{c} x P\left(R_{q}>x\right) d x .
\end{aligned}
$$

In this right-hand side, use the left-hand equality in (5.6) on the first term, use the right-hand equality and the extended Fatou Lemma on the second term, and use the left-hand equality and Fatou's Lemma on 
the third term, to give

$$
\begin{gathered}
\liminf _{q \rightarrow \infty} E\left(R_{q}^{2}\right) \geq c^{2} P\left(\alpha^{-1} Z>-c\right)+2 \int_{-c}^{0} x P\left(1+\alpha^{-1} Z>x\right) d x \\
+2 \int_{0}^{c} x P\left(\alpha^{-1} Z>x\right) d x .
\end{gathered}
$$

By two integrations by parts this becomes

$$
\begin{array}{rl}
\liminf _{q \rightarrow \infty} E\left(R_{q}^{2}\right) \geq c^{2} & P\left(\alpha^{-1} Z>-c\right)-c^{2} P\left(1+\alpha^{-1} Z>-c\right) \\
& +E\left(\left(1+\alpha^{-1} Z\right)^{2} \mathbf{1}_{-c<1+\alpha^{-1} Z \leq 0}\right) \\
& +c^{2} P\left(\alpha^{-1} Z>c\right)+E\left(\left(\alpha^{-1} Z\right)^{2} \mathbf{1}_{0<\alpha^{-1} Z \leq c}\right) \\
=- & c^{2} P\left(-c-1<\alpha^{-1} Z \leq-c\right) \\
& +E\left(\left(1+\alpha^{-1} Z\right)^{2} \mathbf{1}_{-c<1+\alpha^{-1} Z \leq 0}\right) \\
& +c^{2} P\left(\alpha^{-1} Z>c\right)+E\left(\left(\alpha^{-1} Z\right)^{2} \mathbf{1}_{0<\alpha^{-1} Z \leq c}\right) \\
\geq- & c^{2} P\left(\alpha^{-1} Z \leq-c\right)+E\left(\left(1+\alpha^{-1} Z\right)^{2} \mathbf{1}_{-c<1+\alpha^{-1} Z \leq 0}\right) \\
& +E\left(\left(\alpha^{-1} Z\right)^{2} \mathbf{1}_{0<\alpha^{-1} Z \leq c}\right) .
\end{array}
$$

On letting $c \rightarrow \infty$ we obtain the lower bound in (4.3).

Proof of Theorem 4.5. By Lemma 4.4,

$$
\begin{aligned}
\limsup _{q \rightarrow \infty} & E\left(R_{q}^{2}\right) \\
\leq & E\left(\left(\alpha^{-1} Z\right)^{2} \mathbf{1}_{Z \leq 0}\right)+E\left(\left(1+\alpha^{-1} Z\right)^{2} \mathbf{1}_{0<1+\alpha^{-1}} Z \leq 1\right) \\
& \quad+E\left(\left(1+\alpha^{-1} Z\right)^{2} \mathbf{1}_{Z>0}\right) \\
= & E\left(\left(\alpha^{-1} Z\right)^{2}\right)+\theta(\alpha)+P(Z>0)+\frac{2}{\alpha} E\left(Z \mathbf{1}_{Z>0}\right) .
\end{aligned}
$$

Now $R_{q}=N_{q}-b_{q}$, so var $N_{q}=\operatorname{var} R_{q}=E\left(R_{q}^{2}\right)-\left(E R_{q}\right)^{2}$. From (5.5) we have $\liminf _{q \rightarrow \infty} E R_{q} \geq E\left(\alpha^{-1} Z\right)=\gamma / \alpha>0$, so $\liminf _{q \rightarrow \infty}\left(E R_{q}\right)^{2} \geq$ $\left(E\left(\alpha^{-1} Z\right)\right)^{2}$. With $(5.9)$ this gives

$$
\limsup _{q \rightarrow \infty} \operatorname{var} N_{q} \leq \operatorname{var}\left(\alpha^{-1} Z\right)+\theta(\alpha)+P(Z>0)+\frac{2}{\alpha} E\left(Z \mathbf{1}_{Z>0}\right) .
$$

We have $\operatorname{var} Z=\pi^{2} / 6$, while $P(Z>0)=1-e^{-1}$. Also $E\left(Z \mathbf{1}_{Z>0}\right)=$ $\gamma-E\left(Z \mathbf{1}_{Z \leq 0}\right)$, and

$$
\begin{aligned}
-E\left(Z \mathbf{1}_{Z \leq 0}\right) & =\int_{-\infty}^{0}(-z) e^{-z} \exp \left(-e^{-z}\right) d z \\
& =\int_{1}^{\infty}(\log t) e^{-t} d t=\int_{1}^{\infty} \frac{e^{-t}}{t} d t=E_{1}(1) .
\end{aligned}
$$

The bound

$$
\limsup _{q \rightarrow \infty} \operatorname{var} N_{q} \leq \frac{\pi^{2}}{6 \alpha^{2}}+\theta(\alpha)+1-e^{-1}+\frac{2\left(\gamma+E_{1}(1)\right)}{\alpha}
$$


follows.

For the lower bound, the lower bound in Lemma 4.4 may be written

$$
\begin{aligned}
\liminf _{q \rightarrow \infty} E\left(R_{q}^{2}\right) \geq & E\left(\left(1+\alpha^{-1} Z\right)^{2} \mathbf{1}_{Z \leq 0}\right. \\
& \quad-E\left(\left(1+\alpha^{-1} Z\right)^{2} \mathbf{1}_{0<1+\alpha^{-1} Z \leq 1}\right)+E\left(\left(\alpha^{-1} Z\right)^{2} \mathbf{1}_{Z>0}\right) \\
= & E\left(\left(\alpha^{-1} Z\right)^{2}\right)-\theta(\alpha)+P(Z \leq 0)+2 E\left(\alpha^{-1} Z \mathbf{1}_{Z \leq 0}\right) .
\end{aligned}
$$

From (5.5) we have $0<\lim \sup _{q \rightarrow \infty} E R_{q} \leq E\left(1+\alpha^{-1} Z\right)$, so $\lim \sup _{q \rightarrow \infty}$ $\left(E R_{q}\right)^{2} \leq 1+2 E\left(\alpha^{-1} Z\right)+\left(E\left(\alpha^{-1} Z\right)\right)^{2}$, which with the above gives

$$
\begin{aligned}
& \liminf _{q \rightarrow \infty} \operatorname{var} R_{q} \\
& \quad \geq \operatorname{var}\left(\alpha^{-1} Z\right)-\theta(\alpha)-1+P(Z \leq 0)-2 \alpha^{-1}\left(E Z-E\left(Z \mathbf{1}_{Z \leq 0}\right)\right) .
\end{aligned}
$$

Thus, since var $N_{q}=\operatorname{var} R_{q}$,

$$
\liminf _{q \rightarrow \infty} \operatorname{var} N_{q} \geq \frac{\pi^{2}}{6 \alpha^{2}}-\theta(\alpha)-1+e^{-1}-\frac{2\left(\gamma+E_{1}(1)\right)}{\alpha},
$$

which is the required lower bound on $\lim \inf _{q \rightarrow \infty} \operatorname{var} N_{q}$ and completes the proof.

\section{NumeriCAL RESULTS}

Matlab and Pascal were used to evaluate $E N_{q}$ for different values of $a$ and $q$. Fig. 1 shows values for $E N_{q}$ for different values of $a$ for tests with up to 20 questions. For example, for a test with 10 alternatives for each question $E N_{q}$ ranges from 29 when there is one question in the test to only 56 when there are 20 questions. Contrast this with the total number of possible tests, which increases from 10 to $10^{20}$ in this range.

These results led the authors to extend the investigation to consider tests containing up to 200 questions. Fig. 2 demonstrates that, as the number of questions in a test is increased, the average number of tests required in order for all possible questions to have appeared increases quite slowly. In a 200-question test with 10 alternatives for each question, there are $10^{200}$ different possible tests and a total bank of 2000 questions; however, on average all questions will have appeared at least once by the time only 78 tests have been generated. The table below summarises the results from Fig. 2, giving $E N_{q}$ for different values of $a$ and $q$.

\begin{tabular}{c|ccccccc} 
Number of alternatives & \multicolumn{8}{|c}{ Number of questions in test $(q)$} \\
for each question $(a)$ & 1 & 5 & 10 & 20 & 50 & 100 & 200 \\
\hline 5 & $11 \cdot 4$ & $17 \cdot 8$ & $20 \cdot 8$ & $23 \cdot 8$ & $27 \cdot 9$ & $31 \cdot 0$ & $34 \cdot 1$ \\
10 & $29 \cdot 3$ & $43 \cdot 5$ & $49 \cdot 9$ & $56 \cdot 4$ & $65 \cdot 0$ & $71 \cdot 6$ & $78 \cdot 1$ \\
20 & $72 \cdot 0$ & $102 \cdot 0$ & $115 \cdot 3$ & $128 \cdot 7$ & $146 \cdot 5$ & $160 \cdot 0$ & $173 \cdot 5$
\end{tabular}




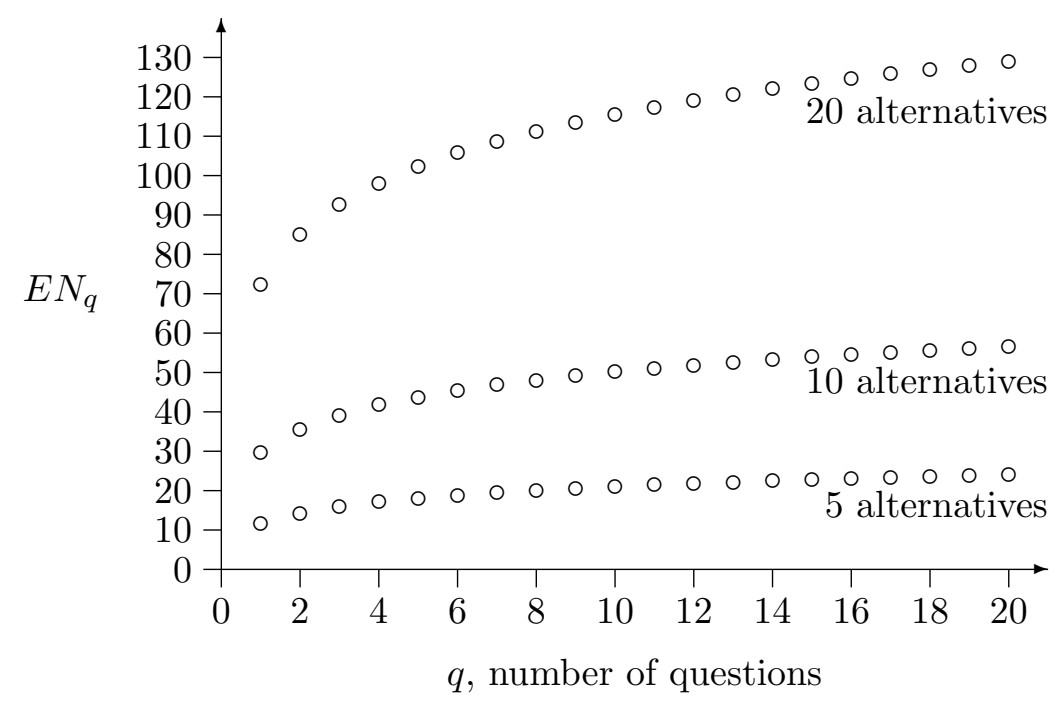

FiguRE 1. $E N_{q}$, the expected number of tests that need to be generated in order for all questions to have appeared at least once, for tests with up to 20 questions and 5, 10, and 20 alternatives for each question

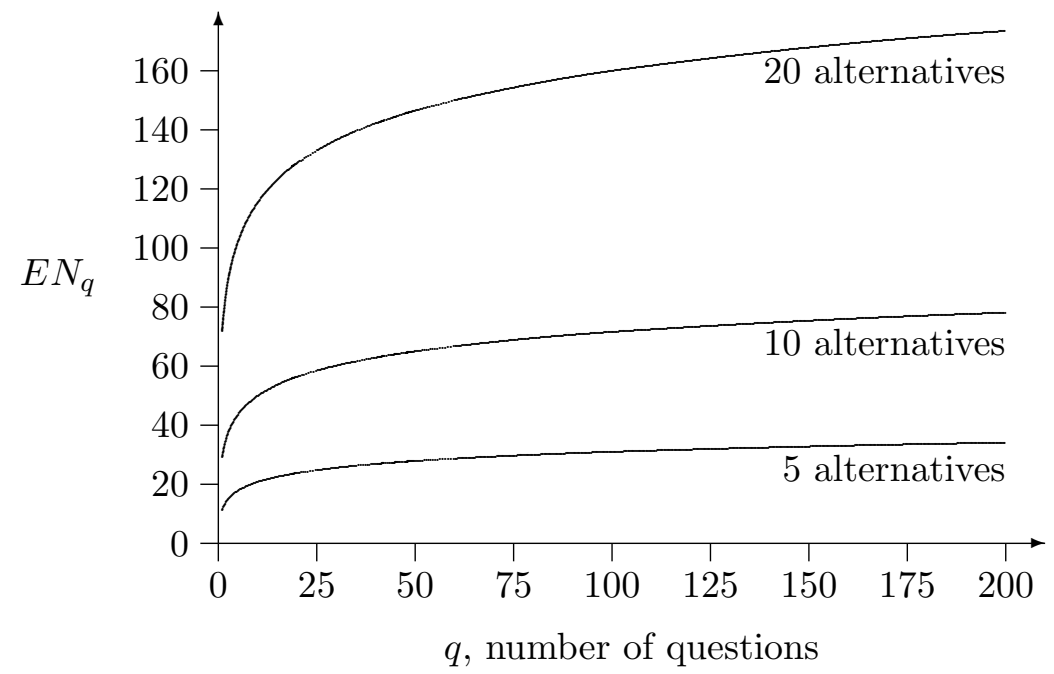

Figure 2. $E N_{q}$, the average number of tests that need to be generated in order for all questions to have appeared at least once, for tests with up to 200 questions and 5, 10, and 20 alternatives for each question

\section{Discussion}

The asymptotics concern the behaviour of the random variable $N_{q}$, defined in $\S 3$, as the number of questions, $q$, grows. There is also dependence on $a$, the number of alternative answers per question in 
the multiple choice, but we regard $a$ as fixed; it is any integer at least 2 , and we set

$$
\alpha:=\log \left(\frac{a}{a-1}\right),
$$

so $\alpha>0$. Theorem 4.1 first says that $N_{q}$ cannot be centred and normed so that its distribution properly converges (one could get convergence to 0, of course, just by heavy norming). However it then says that by centring (translation) alone, $N_{q}$ comes very close to looking like the random variable $Z / \alpha$, where $Z$ has the Gumbel distribution. The difference is a 'wobble' of between 0 and 1 in the limit; persistence of discreteness is responsible for this.

Theorem 4.3 establishes that the expected value of $N_{q}$ behaves accordingly, growing like $\alpha^{-1} \log q$. More exactly, after centring by $b_{q}:=$ $\alpha^{-1} \log (a q)$ it differs from $\alpha^{-1} E Z$ by a number between 0 and 1 in the limit. The table below gives values of $b_{q}+\alpha^{-1} E Z$ for different values of $a$ and $q$.

\begin{tabular}{c|ccccccc} 
Number of alternatives & \multicolumn{8}{|c}{ Number of questions in test $(q)$} \\
for each question $(a)$ & 1 & 5 & 10 & 20 & 50 & 100 & 200 \\
\hline 5 & $9 \cdot 8$ & $17 \cdot 0$ & $20 \cdot 1$ & $23 \cdot 2$ & $27 \cdot 3$ & $30 \cdot 4$ & $33 \cdot 5$ \\
10 & $27 \cdot 3$ & $42 \cdot 6$ & $49 \cdot 2$ & $55 \cdot 8$ & $64 \cdot 5$ & $71 \cdot 0$ & $77 \cdot 6$ \\
20 & $68 \cdot 7$ & $101 \cdot 0$ & $114 \cdot 5$ & $128 \cdot 1$ & $145 \cdot 9$ & $159 \cdot 4$ & $173 \cdot 0$
\end{tabular}

For $q \geq 20$ the actual values of $E N_{q}$ in the previous table exceed these by $0 \cdot 5-0 \cdot 6$, exactly as Theorem 4.3 predicts.

What about the variance of $N_{q}$ as $q$ grows? Theorem 4.5 says that it does not tend to infinity, but is trapped as $q \rightarrow \infty$ between bounds that do not depend on $q$. The precision is pleasing, given that $N_{q}$ does not converge, in any sense. The asymptotic bounds on the variance of $N_{q}$, var $N_{q}$, are $\frac{\pi^{2}}{6 \alpha^{2}} \pm \Delta$ where $\Delta$ is a strange jumble of constants:

$$
\Delta=\theta(\alpha)+1-e^{-1}+\frac{2\left(\gamma+E_{1}(1)\right)}{\alpha}
$$

(the bounds are not claimed to be sharp).

The amount of variability can be better appreciated through the standard deviation. The asymptotic bounds on the standard deviation of $N_{q}$ are

$$
\sqrt{\frac{\pi^{2}}{6 \alpha^{2}} \pm \Delta}
$$

and some values for these are below.

\begin{tabular}{l|cccccc}
$a$ & 2 & 3 & 4 & 5 & 10 & 20 \\
\hline Min s.d. & $0 \cdot 641$ & $2 \cdot 323$ & $3 \cdot 697$ & $5 \cdot 024$ & $11 \cdot 507$ & $24 \cdot 362$ \\
Max s.d. & $2 \cdot 537$ & $3 \cdot 823$ & $5 \cdot 107$ & $6 \cdot 390$ & $12 \cdot 804$ & $25 \cdot 630$
\end{tabular}

The lower bound is non-trivial, i.e. positive, in each case. 


\section{Acknowledgements}

We are grateful to Dave Pidcock, a colleague in the Mathematics Education Centre at Loughborough University, for raising the query in the first place. As a member of staff using computer-based tests to assess students, he was concerned about this issue from a practical viewpoint. That led $\mathrm{RC}$ to post a query on Allstat. CMG was not the only person to respond to the query, and we also acknowledge the others who responded, particularly Simon Bond.

\section{REFERENCES}

[1] M. Abramowitz and I. A. Stegun (ed.), Handbook of Mathematical Functions. Dover, New York, 1965.

[2] I. Adler and S. M. Ross, 'The coupon subset collection problem', J. Applied Probability 38(2001), 737-746.

[3] C. W. Anderson, 'Extreme value theory for a class of discrete distributions with applications to some stochastic processes', J. Applied Probability 7(1970), 99-113.

[4] C. W. Anderson, 'Local limit theory for the maxima of discrete random variables', Math. Proc. Camb. Phil. Soc. 88(1980), 161-165.

[5] R. Cornish, C. M. Goldie and C. L. Robinson, 'Computerassisted assessment: how many questions are enough?', ComputerAided Assessment in Mathematics (February 2006), 9pp., http://mathstore.ac.uk/articles/maths-caa-series/feb2006.

[6] W. Feller, An Introduction to Probability and its Applications, vol. 1, 3rd ed., Wiley, New York, 1968.

[7] G. R. Grimmett and D. R. Stirzaker, Probability and Random Processes, 3rd ed., Oxford Univ. Press, 2001.

[8] J. E. Littlewood (ed. B. Bollobás), Littlewood's Miscellany, Cambridge Univ. Press, 1986.

[9] S. I. Resnick, Extreme Values, Regular Variation, and Point Processes, Springer-Verlag, New York, 1987.

Mathematics Department, Mantell Building, University of Sussex, BRIGHTON BN1 9RF, UK

E-mail address: C.M.Goldie@sussex.ac.uk

Department of Social Medicine, University of Bristol, Canynge Hall, 39 Whatley Road, Bristol BS8 2PS, UK

E-mail address: R.Cornish@bristol.ac.uk

Mathematics Education Centre, Schofield Building, Loughborough

University, Loughborough LE11 3TU, UK

E-mail address: C.L.Robinson@lboro.ac.uk 